\title{
Consideration of enabling and enterprise functions within Defence force design
}

\author{
$\underline{\text { J. Boyce }}^{\text {a }}$, N. Tay ${ }^{\text {a }}$, C. Rowe ${ }^{\text {a }}$ \\ ${ }^{a}$ Defence Science and Technology Group, Canberra, Australian Capital Territory \\ Email:jake.boyce@dst.defence.gov.au
}

\begin{abstract}
Defence force design is the process for planning and designing the future Australian Defence Force (ADF) and its supporting organisation. Traditionally, force design has focussed on major equipment acquisitions such as ships or aircraft, not least because of the long lead time for delivery of such assets. However, there is a need for a coherent force design process that provides better long term planning for the enabling and supporting functions, in addition to the warfighting functions. This paper describes the novel application of a prioritisation method to assess investment options relating to supporting, enabling and business functions of Defence, which are collectively described as enterprise functions. These enterprise functions include, for example, capability acquisition, facility management, finance, military strategy, force design, science and technology, and the engagement functions with the rest of the Australian Government.
\end{abstract}

The Australian Department of Defence operates a biannual cycle called the Defence Capability Assessment Program (DCAP), which is the analytical process that assesses strategic guidance and determines organisational design and investment priorities to achieve the Defence strategy. The various stages of the DCAP include collating gaps and opportunities, prioritising needs and developing force options, then assessing and prioritising these options. Selected options are included in the Integrated Investment Program (IIP) in order to maximise the overall benefit to Defence for a given budget across the next 20 years. DCAP has historically focussed on major equipment acquisitions, but the 2020 Force Structure Plan stated that Defence would return to government with a detailed proposal for workforce growth, which was then addressed within the DCAP 2020-21. This was the first DCAP with a primary focus on workforce.

The DCAP is based on a Capability Based Planning (CBP) approach, which is best suited to assess the operational components of Defence that contribute directly to a warfighting scenario. However, it is conceptually difficult to apply the same approach to the enterprise force options. For example, is it possible to estimate the contribution of a pay clerk to the ADF defeating an adversary in battle? DCAP 2020-21 investigated innovative ways to assess 'enterprise' force options to improve these functions in parallel with the 'operational' force options, which were assessed through a CBP approach.

The Best Worst Method was selected as the most robust, suitable prioritisation tool to assess the enterprise options. Representatives from every Group and Service of Defence were asked to generate a narrative of how each of their proposed force options mitigates risk to Defence objectives, including the targets set out in the Defence Corporate Plan. Each Group and Service then used these narratives to select their most-valuable (best) and least-valuable (worst) force options and to make a relative comparison of every other option against these best and worst. These pairwise comparisons were converted to a quantitative value score for each option. The individual lists were displayed in a visual 'stitching' tool that allowed linear scaling and translation of each list to adjust their position on a common scale. Facilitated discussion amongst the representatives generated an agreed list of the relative values of every option. These enterprise option scores were then stitched together with the operational scores for input to a portfolio optimisation tool and generate proposed portfolios of investment.

This process demonstrated one approach to the treatment of supporting and enabling functions that complements the assessment of warfighting capabilities. When presented to senior decision makers, the enterprise scores were accepted as a fair representation of the relative value of the proposed options. The output of DCAP 2020-21 will underpin both the updated IIP and the Defence workforce growth options developed in response to the 2020 Force Structure Plan.

Keywords: Force design, capability development, decision making, organisational design 


\section{INTRODUCTION}

Force design is the process for planning and designing the Australian Defence Force (ADF) and its supporting organisation. The Australian Department of Defence operates a biannual cycle called the Defence Capability Assessment Program (DCAP), which is the analytical process that assesses strategic guidance and determines organisational design and investment priorities to achieve the Defence strategy (Dept of Defence 2020a). DCAP comprises eight stages that collate gaps and opportunities, analyse capability shortfalls, prioritise needs and develop investment options for the Defence Integrated Investment Program (IIP) submitted through committees to government (Hupfeld 2017). Following the 2020 Force Structure Plan (Dept of Defence, 2020b), the focus for DCAP 2020-21 was on assessing where additional workforce is best recruited to address shortfalls.

Long-term Defence planning has traditionally addressed major equipment acquisitions such as ships or aircraft, not least because of the long lead time for delivery of such assets. However, the focus on Defence workforce in this DCAP cycle made it more important than ever to consider the supporting and enabling functions of Defence in the force design process. This paper describes the novel application of a prioritisation method to assess options relating to these supporting, enabling and business functions, which are collectively described as 'enterprise' functions. This process used successive stages of multiple evaluations of the Best Worst Method (BWM), developed by Rezaei (2015), and linear stitching of the resultant lists to produce scores for the options submitted by the various parts of the Australian Defence Organisation ${ }^{1}$ (ADO), known as the Groups and Services ${ }^{2}$.

\section{BACKGROUND}

The DCAP is an instantiation of the capability-based planning (CBP) process developed by The Technical Cooperation Program (TTCP), which is a five nation Defence research collaboration. The CBP process was developed to facilitate long-term force structure planning and provide decision-makers with a more rational basis to make decisions on future acquisition and with better planning against uncertainty (e.g. in cost, time and performance) and risk (TTCP 2004). Leung et al (2010) interpret CBP as a two stage process, with the first stage taking an 'outward-looking, strategic, 'top-down' perspective in understanding the demands of the operating environment, the expectations of government and the way the defence force should operate" and the second stage taking an "introspective or 'bottom-up' view to assess the performance of the current system with respect to capability goals."

The first stage of the process is centred around generating the high-level, top-down capability goals. TTCP (2004) states that goal setting should be "determined based on ensuring success (appropriately defined) in the most stressing task facing a capability in a specific scenario. This helps establish the maximum capability level required and reduces the number of goals that have to be developed, and thus measured, for each capability." In addition to the scenarios, the key inputs into these goals are the operational concepts describing how the ADF plans to operate, and capability partitions, which group together selected capabilities in packages that can be assessed more manageably. These goals are then a key input into the second stage where existing and future capability options are assessed. It is this second, bottom-up stage that receives the most emphasis in the Australian DCAP process, which focusses on gaps and opportunities submitted by Groups and Services within the ADO. The eight steps of the DCAP are shown in Figure 1.

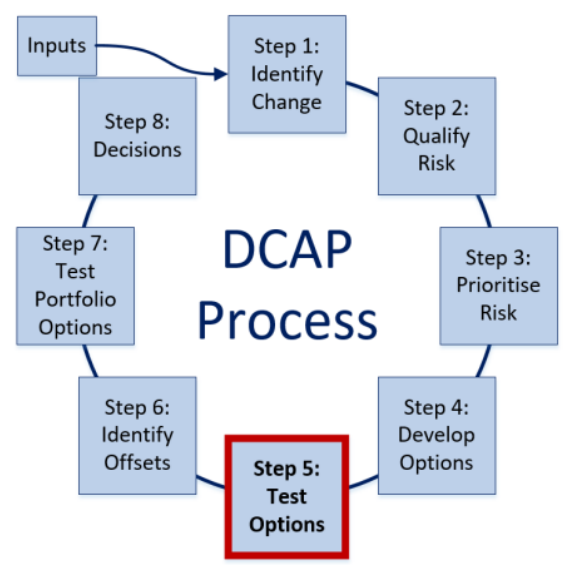

Figure 1. The eight steps of the biannual Defence Capability Assessment Program. This paper proposes a novel approach to assess enterprise options in Step 5.

\section{UNDERSTANDING DEFENCE ENTERPRISE AND ENABLING FUNCTIONS}

In DCAP 2020-21, a wide range of analysis methods were applied to develop a thorough understanding of the likely future needs of the ADO workforce (Rowe et al, 2021). This included modelling of the workforce required to support future scenarios from an operational perspective, as well as achievability analysis to inform

\footnotetext{
${ }^{1}$ The Australian Defence Organisation (ADO) consists of both the ADF and the Department of Defence.

${ }^{2}$ Services are the Royal Australian Navy, Australian Army and Royal Australian Air Force. Groups are other parts of Defence such as the Defence Science and Technology Group or Joint Operations Command.
} 
how quickly the organisation would be able to grow different skill sets, considering the ability to recruit, train, absorb and retain different workforce segments.

CBP is centred on which capabilities best contribute to specific warfighting scenarios. While all elements of the ADO ultimately contribute to military capability, many of the enterprise components are too far removed from delivering military effects for it to be feasible to assess their impact through a scenario-based approach. For example, although the training of new recruits is a vital function, it is difficult to quantify the impact of extra trainers on the chance of winning a specific conflict.

The approach employed for the DCAP 2020-21 cycle required a blend of CBP for operational components and direct assessment of options using multi-criteria decision analysis for the enterprise or business components. The 'operational' components are those that can be meaningfully tested through the operational scenario and this includes a range of enabling functions such as intelligence and information technology infrastructure. The remainder are 'enterprise' components, which are the business functions and other enablers that are too far removed from delivering military effects to enable their inclusion in the scenario. These enterprise functions include capability acquisition, facility management, finance, military strategy, force design, science and technology, and the engagement functions with the rest of the government. By distinguishing between these operational and enterprise subsets of Defence and holistically assessing the requirements for both, Defence has been able to elicit an all-encompassing view of its future workforce and capability requirements.

In mid-2020, all the Groups and Services were asked to identify risks to their capability that could be mitigated through investment in workforce or acquisitions, as well as opportunities for divestment to reallocate resources; these were known as 'gap and opportunity (G\&O)' submissions. In October 2020, a workforce-focussed event was held to refine these G\&O submissions. Firstly, the benefit of addressing each G\&O was assessed through examination of the effect of the additional workforce (or other capability investment) on a complex warfighting scenario - the operational perspective. Secondly, the G\&Os were examined in terms of their impact on the Defence Corporate Plan priorities - the enterprise perspective. This allowed for the G\&Os to be evaluated against the full range of activities that Defence will "undertake to achieve its purpose and to manage enterprise risks" (Dept of Defence 2020c) as well as a warfighting scenario. The evidence from this 2020 event informed a preliminary down-selection of G\&Os by senior Defence officials in a series of workshops, in which G\&Os were classified according to whether they 'must', 'should' or 'could' be addressed in this DCAP cycle.

Following the identification, refinement, and risk qualification of G\&Os, force options were developed to address each G\&O from October 2020 through to the end of February 2021. These force options were either capability options, involving a spend spread for capability acquisition or sustainment, or workforce options, comprised of a number of additional Australian Public Service and/or ADF personnel. Some G\&Os had multiple force options proposed, mitigating risk to different extents at different costs, with only one option to be included in the final portfolio.

\section{FORCE OPTION TESTING}

Step 5 of the DCAP cycle is Force Option Testing (see Figure 1), which entails quantifying the benefit from each of the proposed force options so that they can be prioritised. An activity to understand the benefit to Defence from each option was carried out over four fortnights between March and May 2021. Each fortnight of the activity focussed on a specific theme or capability effect, with the last fortnight specifically focussed on generating a quantitative value score for each force option.

The force options were separated into 'operational' and 'enterprise' options. The majority were workforce, though a few acquisition options were included among both the operational and enterprise sets. The operational options were assessed based on the impact they would have on achieving missions within a given scenario. A Bayesian Reasoning Value Model (BRVM) was used to generate scores from these evaluations directly related to the probability of achieving outcomes in the scenario, which is described by Nguyen et al. (2021).

In contrast, the enterprise force options were assessed using a prioritisation method that compared the relative value of one enterprise option against another. The first step in this process was to develop a narrative around how the option mitigates risk. This narrative underpinned the subsequent assessment and helped to standardise how participants evaluated options. The risk mitigation achieved by each option was described under the following themes:

- Capability delivery

- Enterprise resilience (including business processes, people and data)

- Generation and sustainment of the operational force to achieve the scenario

- Innovation 
Boyce et al., Consideration of enabling and enterprise functions within Defence force design

- Australian industry

- International engagement

- Impact on the Defence Corporate Plan targets

Given that there were around 70 enterprise force options, it was not possible to consistently compare each option with all the others to produce one ordered list. To reduce the cognitive load on performing this prioritisation, smaller lists were first ordered using the BWM, then stitched together, as described in the following sections. A suite of web-based applications called the Knowledge Exploration Node (KEN), developed by the Defence Science and Technology Group, was used to perform this two-step prioritisation.

\subsection{Best Worst Method}

The BWM is a decision-making method developed by Rezaei $(2015,2016)$ that uses pairwise comparisons between options against criteria on a Likert scale to generate a quantitative score for each. In contrast with other prioritisation methods such as analytic hierarchy process, BWM works well for larger lists as the number of comparisons scales linearly with the number of options and the double comparison (since each option is compared with the best and the worst option) means that some level of consistency can be measured.

The assessment of options involves several steps: the best and worst options are chosen; the best option is then compared to every other option; and the same is done for the worst option (Figure 2). In each step, a justification should be given so that the prioritisation reasoning is made explicit. The two rounds of qualitative comparisons ensure the scoring is justified, improve granularity beyond the 5 point Likert scale, and produce a metric that can highlight logical inconsistencies, indicating the need to probe further reasoning.

This method can be repeated for multiple criteria, with the criteria weighted to give an overall value score at the end. However, in our application there were a large number of options that some groups needed to assess, and each group's options primarily addressed different risk themes. For every risk theme to be assessed and then assigned abstract weights, the commonality of the assessments would be reduced. Instead, participants were asked to make a single, combined assessment after considering all the different criteria, both greatly reducing the workload and allowing deeper discussion of the merits of options against specific risk themes when placing lists on a common scale in the next stage.

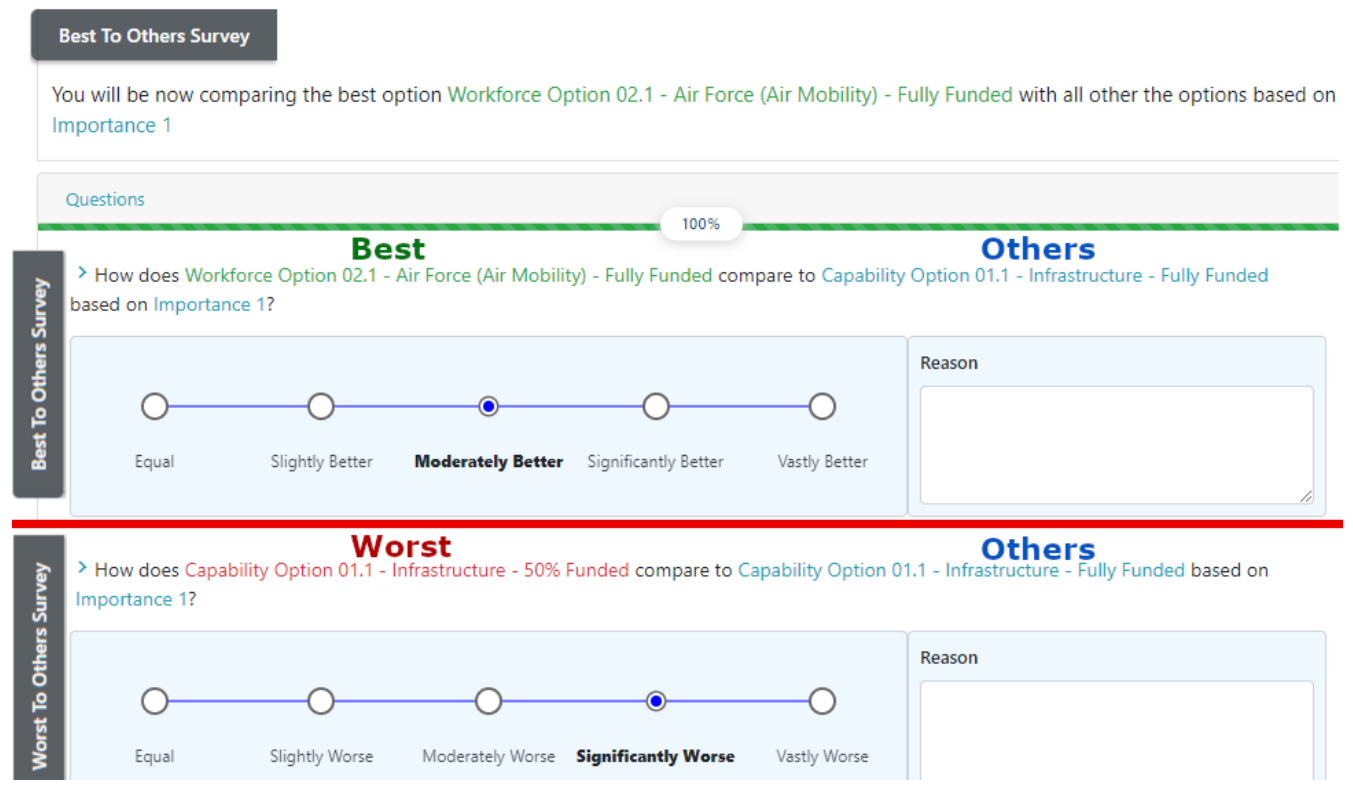

Figure 2. Screenshots of two comparisons in KEN's BWM tool with abstracted force options.

A BWM tool was set up for every Group and Service who had enterprise force options, although some groups were combined into one tool where they only had a very small number. The groups completed the pairwise comparisons, including justification based on the risk mitigation narrative, and the resulting scores were shown in several visualisations (Figure 3) along with the consistency measure; if the group was strongly dissatisfied with the position of certain options or there was high inconsistency, they could edit the evaluation. In Figure 3, the scores are shown along the $\mathrm{x}$-axis, while the $\mathrm{y}$-axis simply indicates where multiple options share a score. 
Boyce et al., Consideration of enabling and enterprise functions within Defence force design

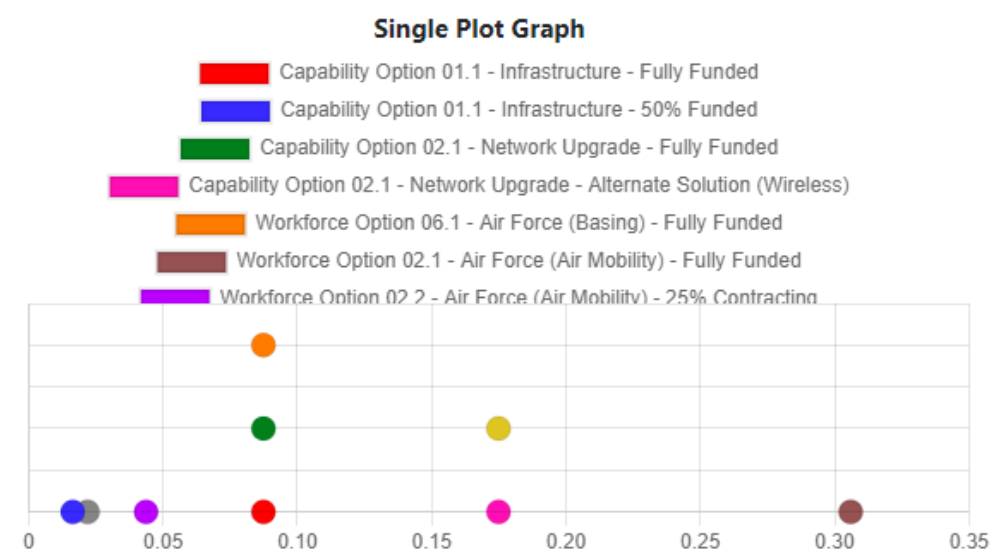

Figure 3. An example visualisation produced by KEN's BWM tool with abstracted force options.

\subsection{Stitching activities}

The Stitching tool within KEN is a visual linear transformation tool that allows multiple scored lists to be stretched and translated by dragging and dropping the top and bottom items in the lists (Figure 4). One advantage of preserving the internal scale of each list and only providing two degrees of freedom is that it reduces the amount of conflict likely to arise between groups who are insistent that one of their options should be higher than another group's option; the top and bottom of each list are reference points that the facilitation can draw the focus to, rather than worrying about the nuances of positioning the options in the middle of the list.

The first three days included between two and five lists to stitch, with each list containing between three and 15 options. On the final day, the three resultant lists were stitched in a facilitated session with one representative from every Group and Service, with the final list containing 68 options.

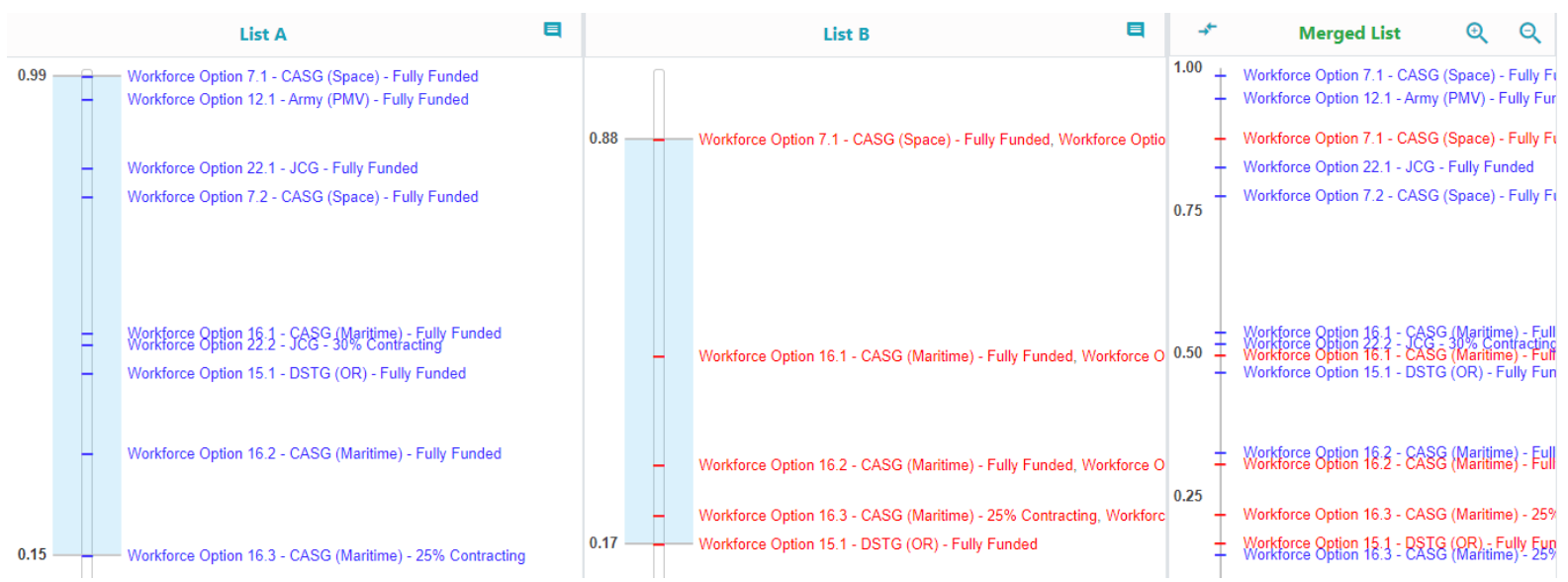

Figure 4. A mock instance of KEN's stitching tool comparing two lists with fabricated force options.

\subsection{Force option value scores as input to subsequent DCAP activities}

The goal of the DCAP cycle is to produce a portfolio of force options that maximises the benefit to Defence for a given budget distributed across the next 20 years. In mathematical language, this is a multiple knapsack problem, requiring the value scores for each force option generated during Force Option Testing. The list of values for operational options generated through BRVM as part of a CBP process were stitched together with the list of enterprise option values elicited via the above described process based on the judgement of senior Defence officials.

A portfolio optimisation tool, NITRO (New Investments to Risk Options), uses Integer Linear Programming (as opposed to a dedicated knapsack algorithm) to "maximise the value of a collection of chosen capabilities subject to remaining within future budget year ceilings" (Calbert et al, 2019). The final combined list of all force options was used to extrapolate values across the 20 year investment period, which were then fed into NITRO to produce a proposed portfolio to be tested further and used to inform the IIP. 
The results of the enterprise assessment, including the risk assessment narratives and value scores, have national security sensitivities and cannot be released.

\section{DISCUSSION}

The application of the BWM of prioritisation was very successful in generating relative values for a very diverse set of investment options. During the whole process, the participants engaged actively in the assessment and discussion. When disagreements in the scores occurred, sufficient time was available for participants to voice their opinions based on their expertise and they usually reached a degree of consensus. While there was not a complete agreement in the final list, when presented to senior decision makers, the enterprise scores were largely accepted as a fair representation of the relative value of the proposed enterprise options.

\subsection{Elicitation issues}

Overall, the facilitation of the activities ran with minimal conflict among participants, despite all having a vested interest in championing particular options. This was managed through the recognition that all force options were important and that the scoring only formed one component of the capability assessment. There were still some aspects that ran less smoothly; for example, the scope of the 'enterprise' portion of the overarching activity was not clear to participants, some of whom were expecting to assess more of their force options, indicating the need for better dissemination of the preliminary option classification.

While it was important to have representatives from each group as Subject Matter Experts (SME) on their force options, some groups have a very broad scope, such as Joint Capabilities Group who had 17 options across domains as diverse as Health and Logistics, so it was unmanageable to include experts on every option. One solution is to promote a shift to centralised G\&O and force option development within Groups and Services so that there is always an SME who can speak to all the options and can be involved through the whole process.

Conflict primarily arose during stitching when options with similar purposes ended up in different areas of the scored list. This only happened a few times, and overall consensus among the participants was reached relatively easily. However, due to the number of options in the final list, once there was satisfaction with reference options being in the correct place it is likely that participants didn't take sufficient time to fully assess the final list and confirm that the majority of options were in reasonable positions.

Options often shared a score due to both the granularity of the stitched list and because BWM gave options the same score if consistency was high due to the five level Likert scale. In addition to difficulties in locating such options, this complicated the stitching facilitation since representatives would disagree that two options were equal when comparing against a broader set of options, insisting that another group's option would sit in between two 'equal' options.

\subsection{Process issues}

Although one benefit of the BWM tool is preventing participants from simply rating options as they like, it is still possible to edit the evaluation after seeing the results, thereby gaming the system. A quirk was also noted when two options were considered equal best: the second option ended up with a higher score than the option chosen as best. Exploration into the mathematics behind this phenomenon is an opportunity for future work.

One potential issue with this process of 10 separate BWM rankings, three intermediary stitchings and one final stitching is that the significance of the distinction between options shrank with each subsequent step, meaning the later steps contributed more to the final distribution of options than the earlier steps. Group A may perceive the top option to be 'vastly better' than the bottom option, but their list may fit in the top half of the day's stitched list, which is then positioned in the top two thirds of the final list: Group A's top option might now have a score around 1.00 while their bottom option is scored 0.66. Further consideration is required to understand whether this is in fact a problem or an expected occurrence when combining diverse options.

This shrinking of the distance between values is one aspect of a problem for the NITRO knapsack optimisation of workforce options. Since NITRO considers value against workforce size (or total cost) to optimise the workforce portfolio, the ratio of value/size is a key metric. This enterprise assessment process generates scores for options between 0.00 and 1.00, and the options were semi-homogenously distributed along a linear scale. However, the workforce sizes of the options spanned up to four orders of magnitude; as such, the value/size metric was dominated by the workforce size in options with extremely small or large workforces. In the example above, Group A's best option is now only $1.5 x$ better than their worst but may cost several orders of magnitude more, making the worst appear to be better value for money. Possible ways considered to remediate this included converting the linear scales into logarithmic/exponential scales, but further investigation is 
required. Another approach to explore is to have the groups rank options both on total size and on the value per unit workforce.

Another issue with the complete CBP process, including the initial capability partitioning and this cycle's separation of options into operational and enterprise, is the potential to overlook the system-level implications. Some interdependencies between options are lost, such as where one option is essential to support another; unless both are included in the portfolio, they may individually be worthless. Similarly, workforce options may be needed to staff capability options. Some of these aspects are assessed during DCAP cycle step 7 - Test Portfolio Options, but an effort should be made to incorporate systems thinking into the problem partitioning.

\section{CONCLUSIONS AND RECOMMENDATIONS}

The DCAP 2020-21 cycle is the first time that Defence has explicitly assessed overarching workforce requirements in the force design process, as well as the first time that distinct assessment processes have been used for the enterprise components of the organisation, separate to the operational components that directly contribute in scenarios. This paper has described the process developed to elicit value scores for the enterprise force options, using the BWM and a linear transform stitching tool, and outlined how this supports subsequent phases of the DCAP. The results of the process have been presented to senior leaders, who considered it a fair representation of the relative value of the proposed enterprise options.

Various aspects of the process require deeper exploration and consideration for the next DCAP cycle, such as propagation of uncertainty from BWM evaluations in the multiple subsequent stitchings and the impact of option size on the prioritisation, including ways to make participants more cognisant of these effects. Additionally, a subsequent activity is required to ensure system-level implications are appropriately considered.

\section{ACKNOWLEDGEMENTS}

The authors would like to thank Joint Experimentation Directorate for the coordination of activities; the KEN team for the development of the tool suite and their assistance; Andrew Coutts and Donald Lowe for program oversight; and John Smith and David Grierson for their facilitation. Thanks also to Russell Connell, Ritchard Walker and David Proctor for managing the facilities that enabled these activities to be conducted.

\section{REFERENCES}

Calbert, G., Weir, T. Garanovich, I. L., Taylor, R., 2019. New Investments to Risk Options (NITRO) - a portfolio analysis and optimisation tool to support force design investment decisions. Paper presented at 23rd International Congress on Modelling and Simulation, Canberra, Australia, December 1-6.

Department of Defence, 2020a. Force Design Guidance, Canberra, Australia.

Department of Defence, 2020b. 2020 Force Structure Plan, Canberra, Australia.

Department of Defence, 2020c. 2020-24 Defence Corporate Plan, Canberra, Australia.

Hupfeld, M., 2017. ADF Force Design and the Capability Life Cycle. Paper presented at 2017 Military Communications and Information Systems Conference, Canberra, Australia, November 14-16.

Leung, C., Nunes-Vaz, R., Prandolini, R., 2010. Capability-Based Planning for Australia's National Security. Security Challenges 6(3), 79-96.

Nguyen, M.-T., et al., 2021. An evaluation model and tool for Defence capability options. In preparation for the 24th International Congress on Modelling and Simulation, Sydney, Australia, December 5-10.

Rezaei, J., 2015. Best-worst multi-criteria decision-making method. Omega 53, 49-57.

Rezaei, J., 2016. Best-worst multi-criteria decision-making method: Some properties and a linear model. Omega 64, 126-130.

Rowe, C., et al, 2021. Analytic Approaches to Understanding Future Defence Workforce Needs. In preparation for the 24th International Congress on Modelling and Simulation, Sydney, Australia, December 5-10.

The Technical Cooperation Program (TTCP), 2004. Guide to Capability-Based Planning. 